
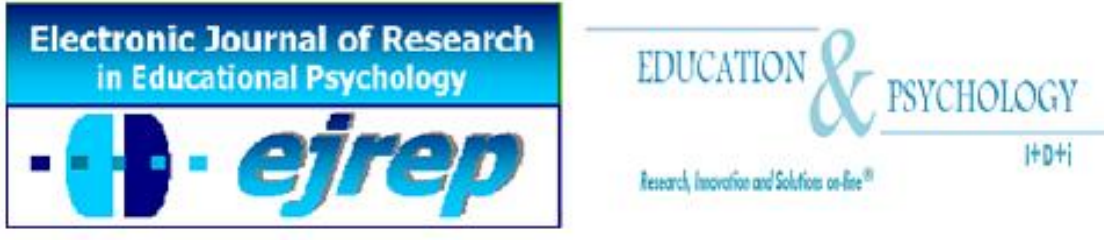

Ilustre Colegio Oficial de Psicología DE ANDALUCIA ORIENTAL

\title{
Inteligencia emocional en alumnado de Educación Secundaria en contextos multiculturales
}

\section{Pegalajar Palomino, $M^{a}$ del Carmen ${ }^{1} \mathbf{y}$ Colmenero Ruiz, $\mathbf{M}^{\mathrm{a}}$ Jesús $^{2}$}

\footnotetext{
${ }^{1}$ Facultad de Ciencias Sociales y de la Comunicación, Universidad Católica de Murcia. Murcia.

${ }^{2}$ Departamento de Pedagogía. Universidad de Jaén. Jaén.
}

\section{España}

Correspondencia: $M^{a}$ del Carmen Pegalajar Palomino. Campus “Los Jerónimos”, s/n. Murcia (Spain). E-mail: mdcpegalajar@ucam.edu

(C) Education \& Psychology I+D+i and Ilustre Colegio Oficial de Psicólogos de Andalucía Oriental (Spain) 


\section{Resumen}

Introducción. Esta investigación pretende analizar el nivel de desarrollo de la inteligencia emocional para alumnado de Educación Secundaria Obligatoria, comprobando la existencia o no de diferencias estadísticamente significativas en función del nivel educativo así como entre alumnado español e inmigrante que asiste al programa de integración Aulas Interculturales Abiertas.

Método. En el trabajo han participado 94 alumnos de un instituto de Educación Secundaria de la provincia de Jaén (España), utilizándose para la recogida de datos un cuestionario de autoinforme sobre educación emocional (Emotional Quotient Inventory: Young Version, EQ-i, YV).

Resultados. Los resultados revelan valores muy altos para cada uno de los factores de la escala, siendo las dimensiones relacionadas con el manejo del estrés y la interpersonal en la que los estudiantes han manifestado menores puntuaciones. De igual modo, se destaca la existencia de diferencias estadísticamente significativas en las valoraciones del alumnado de Educación Secundaria Obligatoria según la procedencia de éste, siendo el alumnado español quién muestra mayores habilidades para entender sus propias emociones y comunicarlas a los otros así como para ejercer un control de las mismas. Finalmente, el análisis de varianza realizado revela la existencia de diferencias estadísticamente significativas entre el alumnado según su nivel educativo; así pues, son los alumnos más jóvenes quiénes muestran unas valoraciones más positivas relacionadas con la dimensión intrapersonal y de manejo del estrés y las emociones.

Discusión y conclusiones. Resulta de vital importancia implementar programas de inteligencia emocional con adolescentes desarrollados en contextos multiculturales. De este modo, y a partir del desarrollo de estas competencias, es como se podrá lograr una sociedad más humana y justa en la que se establezca una auténtica convivencia con ausencia de conflictos.

Palabras Clave: inteligencia emocional, alumnado, inmigración, educación secundaria. 


\title{
Emotional intelligence in Secondary Education Students in Multicultural Contexts
}

\begin{abstract}
Introduction. This study analyzes the level of emotional intelligence development for Secondary Education students. Also, it aims to check statistically significant differences in educational level among Spanish and immigrant students, under the Intercultural Open Classrooms program.
\end{abstract}

Method. The work has involved 94 students of Secondary Education in the province of Jaen (Spain), using for data collection a self-report questionnaire on emotional education (Emotional Quotient Inventory: Young Version, EQ-i: YV).

Results. The results show very high scores for each scale; "Stress management" and "Interpersonal" scales have obtained lower scores. Similarly, there are statistically significant differences in the assessments of Secondary Education students according to their origin; Spanish students show the greatest skills to understand their own emotions and communicate to others and to exercise control over them. The analysis of variance reveals statistically significant differences between students by educational level; younger students show more positive assessments related to "Intrapersonal" and "Stress management" scales.

Discussion and conclusion. It is vital to implement programs that develop emotional intelligence with teenagers in multicultural contexts. Thus, through the development of these skills, it is able to achieve a more humane and just society in which a real life with no conflict is established.

Keywords: emotional intelligence, students, immigration, secondary education. 


\section{Introducción}

El tema de la inteligencia emocional ha destacado en los últimos tiempos al tratarse de una construcción psicológica que presenta una amplia capacidad para explicar el bienestar personal y el éxito social en las organizaciones, la familia y las instituciones educativas. BarOn (2006) lo define como un conjunto interrelacionado de competencias, habilidades y facilitadores emocionales y sociales, que determinan cómo nos entendemos y nos expresamos, cómo entendemos a los demás y nos relacionamos con ellos y nos enfrentamos a las demandas del día a día. Este autor propone quince subescalas para definir la inteligencia emocional; de éstas, diez son consideradas como capacidades básicas y se refieren a aspectos esenciales de la inteligencia emocional (autoconciencia, autoconsideración, asertividad, empatía, relaciones interpersonales, tolerancia al estrés, control del impulso, validación, flexibilidad y solución de problemas) y cinco consideradas como capacidades facilitadoras de la inteligencia emocional (optimismo, autorrealización, alegría, independencia emocional y responsabilidad social). Estas quince subescalas se organizan en cinco grandes dimensiones para el estudio de las características emocionales y sociales de los sujetos tales como:

1) Intrapersonal: habilidad para comprender las propias emociones y su comunicación a los otros.

2) Interpersonal: habilidad para entender y apreciar las emociones de los otros.

3) Manejo de las emociones: habilidad para dirigir y controlar las propias emociones.

4) Adaptabilidad: flexibilidad y eficacia para resolver conflictos.

5) Estado de ánimo general: habilidad para tener una actitud positiva ante la vida.

A día de hoy, la escuela debe hacer frente al desarrollo de las competencias socioemocionales en el alumnado, abarcando desde la protección de los sentimientos, emociones y autoestima hasta la ampliación de sus oportunidades de elegir, reconocimiento de su singularidad, identidad colectiva, personalidad individual y particularidad cultural (Fernández y Terrén, 2008). De este modo, la educación formal y, especialmente, los períodos obligatorios de la enseñanza suponen un momento privilegiado para el desarrollo de las competencias básicas para la convivencia y el establecimiento de relaciones interpersonales (Sanhueza y Cardona, 2009). Su inclusión en el ámbito educativo supone un proceso continuo y gradual de aprendizaje (Dueñas, 2002) el cuál debe partir, necesariamente, de un diagnóstico para poder 
priorizar en las competencias que los estudiantes tienen más deficiencias, sin descuidar el desarrollo global de todas ellas (Soriano y Osorio, 2008).

Más concretamente, la etapa de Educación Secundaria Obligatoria constituye un período crítico para el desarrollo individual y social, en el que los jóvenes se enfrentan a diversas situaciones que evidencian su nivel de desarrollo de inteligencia emocional. Éstos dejan atrás las formas de socialización generalizada de la niñez para relacionarse con sus pares en la búsqueda de comprensión y aceptación social (Zavala, Valadez y Vargas, 2008), siendo uno de los retos más importantes del adolescente el establecer y mantener buenas relaciones con las personas de su entorno (Extremera y Fernández-Berrocal, 2013).

Además, la actual sociedad se caracteriza por la creación de auténticos entornos multiculturales que precisan del conocimiento y respeto de todos y cada uno de los miembros de la comunidad educativa hacia la diversidad de lenguas, modos de vida, comportamientos, sentimientos, emociones, etc. Según Repetto, Pena, Mudarra y Uribarri (2007) el contacto con grupos y etnias dispares obliga al reconocimiento de la multiplicidad de enfoques y, por tanto, al desarrollo de instituciones educativas dialogantes y democráticas centradas en la promoción de la convivencia y en la potenciación de cada uno de los estudiantes y sus enfoques culturales. En este sentido, el programa Aulas Abiertas Interculturales, desarrollado por la Liga Española de la Educación y la Cultura Popular y financiado por el Fondo Social Europeo, pretende lograr la integración socioeducativa del alumnado inmigrante y sus familias en los centros educativos de Educación Primaria y Educación Secundaria Obligatoria, formando grupos heterogéneos que faciliten espacios interculturales de intercambio de vivencias, experiencias y aprendizaje mediante la compensación curricular y las actividades de ocio y tiempo libre basadas en la interculturalidad, la educación en valores y la convivencia.

Fajardo, Patiño y Patino (2008) definen a los adolescentes inmigrantes como un grupo de riesgo al presentar riesgos psicopatológicos asociados a que estos menores experimentan, al mismo tiempo, dos procesos psicológicos: por un lado, la separación de su lugar de origen y aceptación a la nueva cultura y, por el otro, la vivencia de la adolescencia, separación de los viejos apegos emocionales y la tarea de integración de la identidad propia del desarrollo durante esta etapa de ciclo vital. Asimismo, la pertenencia a un grupo socialmente desfavorecido y/o en situación de pobreza, entendida como la convergencia de un déficit económico y una situación de exclusión social (Segura, 2010), puede tener un impacto negativo en la confor- 
mación y expresión de las capacidades sociales (Ayala, Pedroza, Morales, Chaparro y Barragán, 2002). En este sentido, Gualda (2008) hace referencia a la importancia de las redes de amigos para los inmigrantes, pudiendo salir del encapsulamiento familiar y dando lugar a la creación de nuevas alternativas inviables si sólo se contara con redes familiares.

Por ello, la inteligencia emocional se define como un tema tremendamente prometedor aunque resultan escasos los estudios desarrollados en este ámbito con adolescentes ya que, entre otras razones, los instrumentos de que se dispone para acceder al estudio son adaptaciones de instrumentos para adultos o bien, son muy recientes. No obstante, algunas investigaciones establecen relaciones positivas entre la inteligencia emocional y el logro académico, la prevención de conductas de riesgo, el ajuste social y la calidad y cantidad de las relaciones interpersonales (Fernández-Berrocal, Extremera y Palomera, 2008). Además, se ha relacionado la inteligencia emocional con el bienestar subjetivo (Bar-On, 2005), la competencia social (Brasseur y Gregoire, 2011; Zavala, Valadez y Vargas, 2008) y el ajuste socioescolar del alumnado (Pena y Repetto, 2008).

Otros estudios como los desarrollados por Díaz-Aguado y Baraja (1993), León, Felipe, Gómez, Gozalo y Lagas (2007), Rodríguez, Romero, Luque y Rocío (2002) y Siguan (1998) han demostrado cómo los alumnos inmigrantes manifiestan problemas de adaptación social, de competencias sociales y, por tanto, de inclusión social. De igual modo, Fernández, Gaete y Terrén (2008) constataron una integración en el aula significativamente menor entre los estudiantes extranjeros respecto al grupo de españoles, con mayores índices de rechazo y menores niveles de aceptación entre sus compañeros de clase. Finalmente, Giró (2011), citando el Informe "Juventud en España" (2004), señala cómo los adolescentes extranjeros parecen tener un círculo más limitado de amistades que los españoles; los primeros necesitan sentirse "arropados" por personas de la misma edad, pero también de la misma cultura con las que resulta fácil identificarse, existiendo menores grados de confianza con los adolescentes españoles a los que se les considera amigos de circunstancias.

Asimismo, resulta interesante analizar en qué medida influye la edad o el nivel educativo del alumnado en relación a sus valoraciones sobre inteligencia emocional; así, se pueden establecer mecanismos desde la propia institución educativa dirigidos al desarrollo de la educación emocional que "compensen" las deficiencias del alumnado en determinadas edades o etapas educativas. Sin embargo, Chan (2003) comprobó que ni la edad ni el género del alum- 
no ejercían influencia sobre las dimensiones de la inteligencia emocional autopercibida; en esta misma línea, Cuéllar (2012) sostiene cómo no se producen diferencias asociadas a la edad o el género de los alumnos para determinar la relación entre inteligencia interpersonal e intrapersonal y el rendimiento académico en el alumnado. Por su parte, Atkins y Stough (2005) demostraron cómo es más probable que los adultos de mediana edad, al menos, sean capaces de representar y regular emociones más complejas e integrarlas con sus cogniciones sobre el mundo de un modo más experto que los adultos más jóvenes; de este modo, se considera que conforme crecen los niños, aumenta el empleo de estrategias cognitivas frente a las conductuales, pareciéndose cada vez más a las estrategias de afrontamiento de los adultos (Saarni, 2000). No obstante, Álvarez, Bisquerra, Fita, Martínez y Pérez (2000) revelan cómo a medida que aumenta la edad en el niño, tiende a disminuir su nivel de autoestima, siendo a partir de los 14 años cuando más se manifiesta la necesidad de desarrollar programas de educación emocional.

\section{Objetivos}

Teniendo en consideración tales estudios e investigaciones, este trabajo pretende analizar el nivel de desarrollo de la inteligencia emocional para el alumnado de Educación Secundaria Obligatoria, comprobando la existencia o no de diferencias estadísticamente significativas en función del nivel educativo así como entre alumnado español e inmigrante que asiste al programa de integración Aulas Abiertas Interculturales. Para el logro de este objetivo general, se han considerado los siguientes objetivos específicos:

1) Analizar el nivel de desarrollo de la inteligencia emocional en alumnado de Educación Secundaria Obligatoria.

2) Analizar el nivel de desarrollo de la inteligencia emocional entre alumnado español y alumnado inmigrante que asiste al programa de integración Aulas Abiertas Interculturales.

3) Establecer comparaciones en las valoraciones sobre inteligencia emocional según el nivel educativo del alumnado para la etapa de Educación Secundaria Obligatoria. 


\section{Método}

\section{Participantes}

La investigación se ha realizado durante el curso académico 2012/13 en un Instituto de Educación Secundaria Obligatoria de la provincia de Jaén (España). Al albergar un elevado número de alumnado inmigrante extracomunitario, este centro participa en el programa Aulas Abiertas Interculturales para la integración del alumnado inmigrante en la etapa de Educación Secundaria Obligatoria.

La población objeto de estudio está compuesta por 110 estudiantes de Educación Secundaria Obligatoria (entre $1^{\circ}$ y $4^{\circ}$ curso), habiéndose obtenido los resultados mediante un muestreo no probabilístico de tipo accidental o casual (n=94). La tabla 1 detalla la distribución de la muestra según algunas de las características sociodemográficas más relevantes:

Tabla 1. Datos sociodemográficos

\begin{tabular}{|c|c|c|c|}
\hline & & $\mathbf{N}$ & $\%$ \\
\hline \multirow{2}{*}{ Género } & Hombre & 47 & 50.0 \\
\hline & Mujer & 47 & 50.0 \\
\hline \multirow{3}{*}{ Edad } & Menos de 13 años & 10 & 11.0 \\
\hline & Entre 14 y 16 años & 62 & 68.1 \\
\hline & Más de 17 años & 91 & 20.9 \\
\hline \multirow{4}{*}{ Curso } & $1^{\circ} \mathrm{ESO}$ & 25 & 26.5 \\
\hline & $2^{\circ} \mathrm{ESO}$ & 21 & 22.3 \\
\hline & $3^{\circ} \mathrm{ESO}$ & 23 & 24.4 \\
\hline & $4^{\circ} \mathrm{ESO}$ & 25 & 26.5 \\
\hline \multicolumn{2}{|c|}{ Alumnado inmigrante } & 37 & 39.8 \\
\hline \multicolumn{2}{|c|}{ Alumnado español } & 56 & 60.2 \\
\hline
\end{tabular}

\section{Instrumentos}

Para la recogida de datos se ha utilizado el cuestionario de autoinforme sobre inteligencia emocional "Emotional Quotient Inventory Youth Version" (Bar-On y Parker EQ-i:YV, 2000). La principal ventaja de la versión empleada es la duración del tiempo de aplicación de la misma así como la facilidad de la comprensión de las distintas cuestiones por el alumnado. Dicho inventario proporciona información acerca de las competencias sociales y emocionales, permitiendo trazar un perfil social y afectivo (Bar-On y Parker, 2000). Estos autores, además, han informado de la adecuada fiabilidad de las escalas valoradas en el cuestionario, cuyos 
valores oscilan entre .84 (intrapersonal) y .89 (total); por su parte, Ferrándiz, Ferrando, Bermejo y Prieto (2006) confirman una fiabilidad de .87 para el total de la escala a la vez que Prieto, Bai, Ferrandiz y Serna (2007) evidencian una fiabilidad adecuada (.89).

El cuestionario, destinado a alumnado de entre 6 y 18 años, consta de 60 afirmaciones en una escala politómica de cuatro puntos (1=nunca me pasa; 4=siempre me pasa) que valora las siguientes dimensiones:

1) Intrapersonal: habilidad para comprender las propias emociones y su comunicación a los otros;

2) Interpersonal: habilidad para entender y apreciar las emociones de los otros;

3) Manejo de las emociones: habilidad para dirigir y controlar las propias emociones;

4) Adaptabilidad: flexibilidad y eficacia para resolver conflictos;

5) Escala de estado de ánimo general: habilidad para tener una actitud positiva ante la vida.

\section{Procedimiento}

Para la aplicación del cuestionario a la muestra, se ha contactado con la dirección del Instituto de Educación Secundaria y con los responsables del programa Aulas Abiertas Interculturales con la intención de poder explicarles el objetivo de la investigación. Además, se ha contado con el visto bueno de las familias de los estudiantes asegurándole, en todo momento, el anonimato y confidencialidad de los datos recogidos. El cuestionario fue aplicado de manera individual para cada alumno en horario de mañana, facilitándoles el tiempo y las instrucciones necesarias para una adecuada cumplimentación.

\section{Análisis Estadístico}

El tratamiento de los datos ha sido elaborado mediante el programa informático Statistical Package for Social Sciences (SPSS, versión 21 para Windows). Asimismo, se ha realizado un análisis descriptivo de las dimensiones sobre inteligencia emocional incluidas en el cuestionario EQ-i: YV (medias y desviaciones típicas). La prueba $t$ de Student para muestras independientes intenta conocer la existencia o no de diferencias estadísticamente significativas en las valoraciones del alumnado sobre inteligencia emocional según éste sea español o alumnado inmigrante que participa en el programa de integración Aulas Abiertas Intercultura- 
les. Finalmente, y con la intención de conocer la existencia o no de diferencias estadísticamente significativas en las valoraciones del alumnado sobre inteligencia emocional según su nivel educativo, se ha realizado una prueba de comparación de medias mediante el análisis de varianza (ANOVA); además, se ha llevado a cabo un análisis a posteriori mediante la prueba de Tukey a fin de comprobar la diferencia entre todos los pares de medias en el contexto de la muestra total, teniendo en consideración que el contraste está realizado a un 5\%.

\section{Resultados}

El alumnado de Educación Secundaria Obligatoria participante en la investigación obtiene valores muy altos para cada uno de los factores de la escala EQ-i: YV (Tabla 2). Para la dimensión Estado de ánimo, las puntuaciones revelan cómo los estudiantes afirman sentirse felices y aceptarse físicamente, les gusta divertirse y que todo aquello que hacen le salga de manera satisfactoria ( $M=3.04$; DT=.291). La dimensión Adaptabilidad también ha obtenido puntuaciones positivas por parte del alumnado, lo que demuestra que los estudiantes son capaces de encontrar soluciones y resolver situaciones difíciles con facilidad $(\mathrm{M}=2.90$; DT=.392). De igual modo, y en cuanto a la comprensión de las propias emociones y comunicación a los otros (dimensión Intrapersonal), los estudiantes manifiestan conocer sus propios sentimientos y poder expresarlos a los demás $(\mathrm{M}=2.80 ; \mathrm{DT}=.478)$.

Las dimensiones en la que los estudiantes han obtenido menores puntuaciones son las relacionadas con el Manejo del estrés $(\mathrm{M}=2.73$; DT=.415) y la dimensión Interpersonal $(\mathrm{M}=2.72$; $\mathrm{DT}=.346)$. Así pues, en determinadas ocasiones el alumnado presenta problemas para mantenerse tranquilo y adoptar una actitud de calma ante cualquier circunstancia que altere su vida. De igual modo, y sólo a veces, se ven envueltos en situaciones conflictivas para relacionarse con sus amigos y comprender las emociones y sentimientos de los otros.

Tabla 2. Estadísticos descriptivos de los factores de la escala EQ-i: YV

\begin{tabular}{lcccc}
\hline Dimensión & Media & Desviación Típica & Mínimo & Máximo \\
\hline Intrapersonal & 2.80 & $(.478)$ & 1.83 & 3.67 \\
\hline Interpersonal & 2.72 & $(.346)$ & 1.83 & 3.58 \\
\hline Manejo del estrés & 2.73 & $(.415)$ & 1.75 & 3.50 \\
\hline Adaptabilidad & 2.90 & $(.392)$ & 1.80 & 3.70 \\
\hline Estado de ánimo & 3.04 & $(.291)$ & 2.29 & 3.71 \\
\hline
\end{tabular}


A continuación, se ha realizado la prueba $t$ de Student para muestras independientes con la intención de determinar la existencia o no de diferencias estadísticamente significativas en sus valoraciones según éste sea alumnado español o inmigrante y, por tanto, participe en el programa de integración Aulas Abiertas Interculturales (tabla 3).

Así pues, los resultados revelan la existencia de diferencias estadísticamente significativas en las valoraciones del alumnado de Educación Secundaria Obligatoria para la dimensión Intrapersonal $(t(72)=-2.03, p=.04)$ y de Manejo del estrés $(t(70)=-2.18, p=.03)$. No obstante, se descartan la existencia de diferencias estadísticamente significativas en las valoraciones del alumnado de Educación Secundaria Obligatoria que ha participado en la investigación para las dimensiones Interpersonal $(t(78)=1.95, p=.05)$, Adaptabilidad $(t(69)=-.88$, $p=.38)$ y Estado de ánimo $(t(70)=.71, p=.48)$.

Dichas diferencias se decantan a favor del alumnado español quién muestra mayores habilidades para entender sus propias emociones y comunicarlas a los otros así como para ejercer un control de las mismas. Por consiguiente, se destaca cómo el alumnado inmigrante que participa en el programa de integración Aulas Abiertas Interculturales obtiene menores puntuaciones en las dimensiones Intrapersonal y Manejo del estrés y las emociones.

Tabla 3. Prueba t de Student para la escala EQ-i: YV entre alumnado inmigrante y español

\begin{tabular}{lcccc}
\hline & $\begin{array}{c}\text { Alumnado inmigrante } \\
\mathrm{M}(\mathrm{DT})\end{array}$ & $\begin{array}{c}\text { Alumnado español } \\
\mathrm{M}(\mathrm{DT})\end{array}$ & $\mathrm{t}$ & $\mathrm{p}$ \\
\hline Intrapersonal & $2.66(.502)$ & $2.90(.447)$ & -2.06 & $.04^{*}$ \\
\hline Interpersonal & $2.81(.392)$ & $2.65(.303)$ & 1.95 & .05 \\
\hline Manejo del Estrés & $2.60(.444)$ & $2.81(.370)$ & -2.18 & $.03^{*}$ \\
\hline Adaptabilidad & $2.85(.431)$ & $2.94(.358)$ & -.88 & .38 \\
\hline Estado de ánimo & $3.07(.286)$ & $3.02(.297)$ & .71 & .48 \\
\hline \multicolumn{1}{c}{$* p^{\circ .05}$} & & & &
\end{tabular}

Una vez analizadas las valoraciones de los estudiantes de Educación Secundaria sobre inteligencia emocional, resulta interesante determinar entre qué grupos de alumnos se establecen dichas diferencias. Así pues, y para conocer si existen diferencias estadísticamente significativas o no entre el alumnado según el nivel educativo en que se encuentren, se ha realizado un análisis de varianza (ANOVA). Dicho análisis pone de manifiesto la existencia de diferencias estadísticamente significativas para la dimensión Intrapersonal $(F(4,70)=4.05, p=.00) \mathrm{y}$ Manejo del estrés $(F(4,68)=3.88, p=.00)$. No obstante, en las dimensiones Interpersonal 
$(F(4,76)=.47, p=.75)$, Adaptabilidad $(F(4,67)=.97, p=.42)$ y Estado de ánimo $(F(4,67)=.45$, $p=.77)$ no se aprecian diferencias estadísticamente significativas entre los distintos grupos de alumnos (Tabla 4).

Además, la prueba de Tukey realizada a posteriori revela cómo, para la dimensión Intrapersonal, las diferencias se sitúan entre los alumnos de primer y segundo curso y aquellos otros que se encuentran en el último curso de la etapa de Educación Secundaria Obligatoria $(p<.01)$; resultan ser los alumnos más noveles los que presentan un nivel de desarrollo de la inteligencia emocional más favorable. Por su parte, para la dimensión relacionada con el $M a$ nejo del estrés, la prueba de Tukey muestra cómo dichas diferencias se producen entre los alumnos de segundo y tercer curso de Educación Secundaria Obligatoria $(p<.01)$; en este caso, y al igual que para la dimensión anterior, los alumnos más jóvenes muestran unas valoraciones más positivas hacia la inteligencia emocional.

Tabla 4. ANOVA para la escala EQ-i: YV según el nivel educativo del alumnado

\begin{tabular}{lllllll}
\hline & $1^{\circ}$ ESO & $2^{\circ}$ ESO & $3^{\circ}$ ESO & $4^{\circ}$ ESO & \multirow{2}{*}{ ANOVA } & Tukey \\
& $\mathrm{M}(\mathrm{DT})$ & $\mathrm{M}(\mathrm{DT})$ & $\mathrm{M}(\mathrm{DT})$ & $\mathrm{M}(\mathrm{DT})$ & & \\
\hline Intrapersonal & $3.01(.355)$ & $2.88(.437)$ & $2.58(.525)$ & $2.38(.565)$ & $.00^{*}$ & $1^{\mathrm{o}}-4^{\mathrm{o}} ; 2^{\mathrm{o}}-4^{\mathrm{o}}$ \\
\hline Interpersonal & $2.82(.339)$ & $2.69(.342)$ & $2.69(.325)$ & $2.79(.350)$ & .75 & \\
\hline Manejo del estrés & $2.84(.439)$ & $2.84(.422)$ & $2.45(.312)$ & $2.45(.354)$ & $.00^{*}$ & $2^{\circ}-3^{\mathrm{o}}$ \\
\hline Adaptabilidad & $2.83(.497)$ & $2.99(.421)$ & $2.76(.325)$ & $2.80(.447)$ & .42 & \\
\hline Estado de ánimo & $3.15(.386)$ & $3.03(.303)$ & $3.00(.282)$ & $3.02(.271)$ & .77 & \\
\hline$*_{p<.05}$ & & & & & &
\end{tabular}

\section{Discusión y conclusiones}

Esta investigación ha permitido conocer las valoraciones sobre inteligencia emocional del alumnado de Educación Secundaria Obligatoria desarrollado en contextos multiculturales. Además, se ha comprobado la existencia de diferencias estadísticamente significativas en sus valoraciones según el nivel educativo en que éstos se encuentran. Sin duda, un trabajo que ha permitido realizar un diagnóstico de las competencias socioemocionales del alumnado de Educación Secundaria Obligatoria (tanto español como inmigrante) para así establecer hacia qué ámbitos de actuación debe dirigirse la labor docente en el aula (Soriano y Osorio, 2008).

De este modo, resulta positivo el hecho de que los estudiantes de Educación Secundaria Obligatoria encuestados hayan obtenido puntuaciones bastante aceptables en sus valoraciones sobre inteligencia emocional. Dichas habilidades llevan al alumnado hacia la adquisi- 
ción de conocimientos fundamentados sobre las emociones, incidiendo en la motivación y el desarrollo de un clima idóneo para el aprendizaje en el contexto del aula y en el centro, siendo esenciales para la gestión del conflicto y la convivencia escolar (Fernández-Berrocal y Extremera, 2002).

Así pues, el alumnado participante en la investigación muestra una actitud positiva hacia la vida y aquello que le ocurre; adopta criterios basados en la flexibilidad y eficacia para resolver conflictos de la vida diaria a la vez que posee cualidades para comprender las propias emociones y comunicarlas a los otros. No obstante, presenta menores puntuaciones al cuestionar acerca del manejo de sus emociones y sentimientos y comprender la de sus compañeros y personas de su entorno ante determinadas circunstancias. Estos datos hacen que se refuerce la idea de desarrollar programas de educación emocional para la etapa de Educación Secundaria Obligatoria, pues ayuda a favorecer el respeto y la aceptación del "otro" y sus diferencias, así como prevenir y corregir posibles enfrentamientos y conflictos producidos de forma habitual en los centros educativos y en la sociedad en general.

Respecto a las valoraciones sobre inteligencia emocional entre el alumnado español e inmigrante, se observa cómo es el alumnado "autóctono" quién muestra mayores habilidades en el ámbito emocional; así pues, éste afirma comprender y manejar de manera adecuada sus propias emociones y sentimientos. Estos resultados pueden deberse al cambio drástico y, por consiguiente, a la tensión en la que se encuentra el alumnado inmigrante a consecuencia del traslado de un país a otro, el cambio de idioma, de costumbres, de cultura, etc. En este sentido, y refiriéndose a los adolescentes que migran, Feixa (2008) expone cómo éstos no pueden olvidar su pasado, es decir, llegan al lugar de destino con unas identidades personales y sociales condicionadas por su socialización primaria en sus lugares de origen, por las imágenes más o menos idealizadas del lugar de destino y por la experiencia más o menos traumática de la aventura migratoria.

Los resultados obtenidos deben cuestionar la actuación educativa desarrollada en el programa de integración Aulas Abiertas Interculturales, prestando especial atención hacia el desarrollo de las competencias socioemocionales del alumnado para facilitar su bienestar personal y, posteriormente, su inserción en el entorno en que se desenvuelve. De igual modo, las investigaciones de Díaz-Aguado y Baraja (1993), León et al. (2007), Rodríguez et al. (2002) y Siguan (1998) revelan cómo los alumnos inmigrantes manifiestan problemas de adaptación 
social, de competencias sociales y, por tanto, de inclusión social. De igual modo, Fernández, Gaete y Terrén (2008) constataron una integración en el aula significativamente menor entre los estudiantes extranjeros respecto al grupo de españoles, con mayores índices de rechazo y menores niveles de aceptación entre sus compañeros de clase.

Tomando como referencia el nivel educativo del alumnado, se observa cómo los estudiantes de menor nivel (primer y segundo curso de Educación Secundaria Obligatoria) muestran mayores habilidades para la comprensión de sus propias emociones y la comunicación a los demás que aquellos otros que se encuentran en niveles superiores (cuarto curso de Educación Secundaria Obligatoria). De igual modo sucede respecto al manejo de las propias emociones, siendo los estudiantes de segundo curso quiénes muestran mayor habilidad para dirigir sus propios sentimientos que los que se encuentran en tercer curso de Educación Secundaria Obligatoria. Dichas diferencias pueden deberse a los profundos cambios en que se ven envueltos los jóvenes a consecuencia del desarrollo físico, cognitivo, moral, social, psicosexual y afectivo; éstos influyen en su forma de ser y en la manera en que éstos se relacionan con el medio, llegando incluso a experimentar una sensación de consistencia personal y discontinuidad social que contribuye al desarrollo de una crisis de identidad en el adolescente.

Estos resultados guardan relación con los aportados por Álvarez et al. (2000) quiénes revelaron cómo a medida que aumenta la edad del alumnado se manifiesta una mayor necesidad de desarrollar programas de educación emocional. Sin embargo, autores como Chan (2003) o Cuéllar (2012) han demostrado que no se producen diferencias significativas en las valoraciones de los estudiantes sobre inteligencia emocional en función de la edad, mientras que Atkins y Stough (2005) establecieron cómo los adultos de mediana edad presentan mayor capacidad para representar y regular emociones más complejas e integrarlas con sus cogniciones sobre el mundo que los adultos más jóvenes.

En definitiva, centrados en el desarrollo de un proceso de enseñanza y aprendizaje de calidad, resulta de vital importancia implementar programas de inteligencia emocional con adolescentes desarrollados en contextos multiculturales que, de forma explícita, contengan las habilidades emocionales basadas en la capacidad para percibir, comprender y regular las emociones (Grewal y Salovey, 2005). De este modo, y a partir del desarrollo de estas competencias, es como se podrá lograr una sociedad más humana y justa en la que se establezca una auténtica convivencia con ausencia de conflictos. 
No obstante, esta investigación tiene limitaciones. La escasa muestra con la que se ha llevado a cabo esta investigación no permite garantizar la generalización de los resultados a otras muestras. De igual modo, el uso exclusivo de autoinformes como instrumento de recogida de información puede generar en problemas como deseabilidad social y sinceridad. Además, se debe considerar cómo las puntuaciones obtenidas sobre inteligencia emocional en alumnado inmigrante se han obtenido con baremos españoles.

Sin embargo, y a pesar de las referidas limitaciones, no cabe duda de que el estudio plantea una serie de interrogantes que fomentan la continuidad en esta línea de investigación. De este modo, puede resultar interesante planificar el desarrollo de un programa sobre inteligencia emocional para este tipo de alumnado, comparando sus valoraciones sobre inteligencia emocional con las expresadas en este estudio. Además, se podría ampliar la muestra objeto de estudio, analizando el nivel de desarrollo de la inteligencia emocional en alumnado desarrollado en contextos multiculturales para la etapa de Educación Primaria así como también conocer las valoraciones de otros agentes educativos como pueda ser la familia o el propio profesorado del centro educativo.

\section{Referencias}

Álvarez, M., Bisquerra, R., Fita, E., Martínez, F. y Pérez, N. (2000). Evaluación de programas de educación emocional. Revista de Investigación Educativa, 18(2), 587-599.

Atkins, P. y Stough, C. (2005). Does emotional intelligence change with age? Comunicación presentada al Society for Research in Adult Development Annual Conference. Atlanta: GA.

Ayala, H., Pedroza, F., Morales, S., Chaparro, A., y Barragán, N. (2002). Factores de riesgo, factores protectores y generalización del comportamiento agresivo en una muestra de niños en edad escolar. Salud Mental, 25(3), 27-40.

Bar-On, R. (2005). The impact of emotional intelligence and subjetive well-being. Perspectives in Education, 23, 41-61.

Bar-On, R. (2006). The Bar-On model of emotional-social intelligence (ESI). Psicothema, 18, $13-25$.

Bar-On, R. y Parker, J.D.A. (2000). Emotional Quotient Inventory: Youth Version (EQ-i: YV). Technical manual. Canada: Multi-Health Systems. 
Brasseur, S. y Gregoire, J. (2011). L`intelligence émotionnelle - Triz chez les adolescents á haut potential: Spécificités et liens avec la réussite scolaire et les competences socials. Enfance, 62(1), 59-76.

Chan, D.W. (2003). Dimensions of emotional intelligence and their relationships with social coping among gifted adolescents in Hong Kong. Journal of Youth and Adolescence, 32(6), 409-418. doi: 10.1023 / A: 1025982217398

Cuéllar, R. (2012). Relación entre inteligencia emocional y rendimiento académico en alumnos de Educación Primaria. Trabajo Fin de Máster. Universidad Internacional de La Rioja.

Díaz-Aguado, M.J. y Baraja, A. (1993). Interacción educativa y desventaja sociocultural. Madrid: CIDE.

Dueñas, M.L. (2002). Importancia de la inteligencia emocional: un nuevo reto para la orientación educativa. Educación XXI, 5, 77-96. doi: http://dx.doi.org/10.5944/educxx1.5.1.384

Extremera, N. y Fernández-Berrocal, P. (2013). Inteligencia emocional en adolescentes. Padres y Maestros, 352, 34-39. doi: http://dx.doi.org/10.14422/pym.v0i352.1170

Fajardo, M., Patiño, M.I. y Patino, C. (2008). Estudios actuales sobre aculturación y Salud Mental en inmigrantes: Revisión y perspectivas. Revista Iberoamericana de Psicología:: Ciencia y Tecnología, 1, 39-50.

Feixa, C. (2008). Generación Uno Punto Cinco. Revista de Estudios de Juventud. 80, 115 127.

Fernández-Berrocal, P. y Extremera, N. (2002). La inteligencia emocional como una habilidad esencial en la escuela. Revista Iberoamericana de Educación, 29, 1-6.

Fernández, M. y Terrén, E. (2008). De inmigrantes a minorías: temas y problemas de la multiculturalidad. Revista de Educación, 345, 15-21.

Fernández, M., Gaete, J.M. y Terrén, E. (2008). ¿FFronteras en las aulas? Contacto transcultural y endogamia en las interacciones del alumnado. Revista de Educación, 345, 157181.

Fernández-Berrocal, P., Extremera, N. y Palomera, R. (2008). Emotional intelligence as a crucial mental ability on educational context. En A. Valle, J.C., Núñez, R.G., Cabanach, R.G., González-Pineda, J.A. y Rodríguez, S. (Eds.). Handbook of Instructional Resources and Their Applications in the classroom (pp. 67-88). Madrid: Nova. 
Ferrándiz, C., Ferrando, M., Bermejo, M.R. y Prieto, M.D. (2006). Emotional intelligence and personality. Comunicación presentada al British Educational Research Association (BERA). Warwick University.

Giró, J. (2011). Las amistades y el ocio de los adolescentes, hijos de la inmigración. Papers, 96(1), 77-95.

Grewal, D. y Salovey, P. (2005). Feeling smart: The science of emotional intelligence. American Scientist, 93, 330-339.

Gualda, E. (2008). Identidades, autoidentificaciones territoriales y redes sociales de adolescentes y jóvenes inmigrantes. Portularia, Revista de Trabajo Social, 8(1), 111-129.

León, B., Felipe, E., Gómez, T., Gozalo, M. y Lagas, C. (2007). Socialización y autoconcepto en una muestra de alumnos inmigrantes marroquíes. Apuntes de Psicología, 25(1), 5365.

Pena, M. y Repetto, E. (2008). Estado de la investigación en España sobre inteligencia emocional en el ámbito educativo. Electronic Journal of Research in Educational Psychology, 6(2), 400-420.

Prieto, M.D., Bai, L., Ferrandiz, C. y Serna, B. (2007). Psychometric Characteristics of EQi:YV in an English sample. Comunicación presentada al I Congreso Internacional de Inteligencia Emocional. Málaga.

Repetto, E., Pena, M., Mudarra, M.J. y Uribarri, M. (2007). Orientación de las competencias socioemocionales de los alumnos de Educación Secundaria en contextos multiculturales. Electronic Journal of Research in Educational Psychology, 5(1), 159-178.

Rodríguez, G., Romero, J.F., Luque, D. y Rocío, L. (2002). Integración escolar y rendimiento de una muestra de alumnos inmigrantes de Algeciras. Psicología Educativa, 8, 5-20.

Saarni, C. (2000). Emotional Competence. A Developmental Perspective. En R. Bar-On y J.D.A. Parker (Eds.). The Handbook of Emotional Intelligence. Theory, Development, Assessment and Aplication at Home, School and in the Workplac (pp. 68-91). San Francisco, Ca: Jossey-Bass.

Sanhueza, S.V. y Cardona, M.C. (2009). Evaluación de la sensibilidad intercultural en alumnado de Educación Primaria escolarizado en aulas culturalmente diversas. Revista de Investigación Educativa, 27(1), 247-262.

Segura, P. (2010). Pobreza y exclusión social. Diagnóstico de los distritos 4 y 5 de Melilla. Melilla: Ministerio de Trabajo e Inmigración.

Siguán, M. (1998). La escuela y los inmigrantes. Barcelona: Paidós Ecuador. 
Soriano, E. y Osorio, M.M. (2008). Competencias socioemocionales del alumnado "autóctono" e inmigrante en educación secundaria. Bordón, 60(1), 129-148.

Zavala, M.A., Valadez, M.D. y Vargas, M.C. (2008). Inteligencia emocional y habilidades sociales en adolescentes con alta aceptación social. Electronic Journal of Research in Educational Psychology, 6(2), 319-338. 